ANNALES

POLONICI MATHEMATICI

XLIII (1983)

\title{
On full derivatives
}

\author{
by KRYSTYNa SKóRniK (Katowice)
}

In papers [2]-[4] J. Mikusiński introduced the notion of a full derivative and listed certain properties, theorems and comments concerning full derivatives. Functions considered in those papers are defined on subsets of the $q$-dimensional Euclidean space $R^{q}$; their values are real numbers or elements of a Banach space. In [2] and [4] J. Mikusiński stated the following

THEOREM. If the full derivatives $f_{i}=f^{\left(e_{i}\right)}$ and $f_{j}=f^{\left(e_{j}\right)}$ exist in a neighbourhood of a point $x_{0}$ and if, moreover, the full derivatives $f_{i}^{\left(e_{j}\right)}\left(x_{0}\right)$ and $f_{j}^{\left(e_{i}\right)}\left(x_{0}\right)$ exist at $x_{0}$, then they are equal.

This theorem is proved by applying the mean value theorem in a real domain. But this method fails, if the values of functions are in an arbitrary Banach space. We therefore must look for another proof. It can be based on a certain theorem about mappings in Banach spaces.

Let $f$ be a function defined in the $q$-dimensional Euclidean space and taking values in a fixed Banach space. Let $e_{i}$ denote the point whose $i$-th coordinate is 1 and the remaining ones are 0 . If a point $x$ has coordinates $\xi_{1}, \ldots, \xi_{q}$, then the point $x+e_{i} \lambda$ differs from $x$ only by the $i$-th coordinate which is equal to $\xi_{i}+\lambda$.

We say (see [2]) that $u$ is the $i$-th full derivative of $f$ at $x_{0}$, if for each $\varepsilon>0$ there is a neighbourhood $V$ of $x_{0}$ such that

$$
\frac{\left\|f(x)-f(y)-u\left(x_{0}\right) e_{i}(x-y)\right\|}{|x-y|}<\varepsilon
$$

for any points $x, y \in V$ differring from each other by their $i$-th coordinates, and only by those coordinates. (The inner product $e_{i}(x-y)$ is equal to the difference of the $i$-th coordinate of $x$ and $y$.) In other words, the points $x$ and $y$ belong to the intersection of $V$ with the straight line (not necessarily passing through $x_{0}$ ) parallel to the $i$-th coordinate axis.

In the proof of the Theorem we shall use the following

Lemma ([1], Theorem 8.6.2). Let $E$ and $F$ be two Banach spaces, let 
$a, b \in E$ and let $f$ be a differentiable mapping of an open neighbourhood $A$ of the segment $S$ joining $a$ and $b$ into. $F$. Then, for each $x_{0} \in A$, we have

$$
\left\|f(b)-f(a)-f^{\prime}\left(x_{0}\right)(b-a)\right\| \leqslant\|b-a\| \sup _{x \in S}\left\|f^{\prime}(x)-f^{\prime}\left(x_{0}\right)\right\| .
$$

Proof of Theorem. Since $f_{i}$ exists in a neighbourhood of $x_{0}$, there exists, for any $\varepsilon>0$, a ball $K_{r}$ with centre at $x_{0}$ and radius $r>0$, such that

$$
\left\|f(x)-f(y)-f_{i}\left(x_{0}\right) e_{i}(x-y)\right\|<\frac{1}{2} \varepsilon|x-y|
$$

holds for any two points $x, y \in K_{r}$ which differ from each other by their $i$-th coordinates only. Consider the function

$$
g(\xi)=f\left(x_{0}+\left(\xi e_{i}+e_{j}\right) \lambda\right)-f\left(x_{0}+\xi e_{i} \lambda\right),
$$

where $\xi \in[0,1]$ and $|\lambda| \leqslant \frac{1}{2} r, \lambda \neq 0$. We have

$$
g^{\prime}(\xi)=\left[f_{i}\left(x_{0}+\left(\xi e_{i}+e_{j}\right) \lambda\right)-f_{i}\left(x_{0}+\xi e_{i} \lambda\right)\right] \lambda .
$$

Since $f_{i}^{\left(e_{j}\right)}$ exists in a neighbourhood of $x_{0}$, there exists, for any $\varepsilon>0$, a radius $r^{\prime} \leqslant r$ such that for $|\lambda| \leqslant \frac{1}{2} r^{\prime}$ the following inequality holds:

$$
\left\|f_{i}\left(x_{0}+\left(\xi e_{i}+e_{j}\right) \lambda\right)-f_{i}\left(x_{0}+\xi e_{i} \lambda\right)-f_{i}^{\left(e_{j}\right)}\left(x_{0}\right) \lambda\right\| \leqslant \frac{1}{2} \varepsilon|\lambda|,
$$

because the points $x_{0}+\left(\xi e_{i}+e_{j}\right) \lambda, x_{0}+\xi e_{i} \lambda$ differ only by their $j$-th coordinates and belong to the ball $K_{r}$, with centre at $x_{0}$ and radius $r^{\prime}$. Hence by (2) we obtain

$$
\left\|g^{\prime}(\xi)-f_{i}^{\left(e_{j}\right)}\left(x_{0}\right) \lambda^{2}\right\| \leqslant \frac{1}{2} \varepsilon|\lambda|^{2} \quad \text { for } \xi \in[0,1],|\lambda| \leqslant \frac{1}{2} r^{\prime} .
$$

Applying the triangle inequality for norms, Lemma and inequality (3) we have

$$
\begin{aligned}
\left\|g(1)-g(0)-f_{i}^{\left(e_{j}\right)}\left(x_{0}\right) \lambda^{2}\right\| & \leqslant\left\|g(1)-g(0)-g^{\prime}\left(\xi_{0}\right)\right\|+\left\|g^{\prime}\left(\xi_{0}\right)-\lambda^{2} f_{i}^{\left(e_{j}\right)}\left(x_{0}\right)\right\| \\
& \leqslant \sup _{\xi \in[0,1]}\left\|g^{\prime}(\xi)-g^{\prime}\left(\xi_{0}\right)\right\|+\frac{1}{2} \varepsilon \lambda^{2} \leqslant \frac{3}{2} \varepsilon \lambda^{2} .
\end{aligned}
$$

Since the difference

$$
g(1)-g(0)=f\left(x_{0}+\left(e_{i}+e_{j}\right) \lambda\right)-f\left(x_{0}+e_{i} \lambda\right)-f\left(x_{0}+e_{j} \lambda\right)+f\left(x_{0}\right)
$$

is symmetric with respect to $e_{i}$ and $e_{j}$, we have

$$
\left\|g(1)-g(0)-f_{j}^{\left(e_{i}\right)}\left(x_{0}\right) \lambda^{2}\right\| \leqslant \frac{3}{2} \varepsilon \lambda^{2} .
$$

The last two inequalities and the triangle inequality imply

$$
\left\|f_{i}^{\left(e_{j}\right)}\left(x_{0}\right) \lambda^{2}-f_{j}^{\left(e_{i}\right)}\left(x_{0}\right) \lambda^{2}\right\| \leqslant 3 \lambda^{2} \varepsilon \quad \text { for }|\lambda| \leqslant \frac{1}{2} r^{\prime} .
$$


Hence

$$
\left\|f_{i}^{\left(e_{j}\right)}\left(x_{0}\right)-f_{j}^{\left(e_{i}\right)}\left(x_{0}\right)\right\| \leqslant 3 \varepsilon .
$$

Since $\varepsilon$ can be taken arbitrarily small, it follows that

$$
f_{i}^{\left(e_{j}\right)}\left(x_{0}\right)=f_{j}^{\left(e_{i}\right)}\left(x_{0}\right) \text {. }
$$

\section{References}

[1] J. Dieudonne, Foundations of modern analysis, Academic Press, New York and London 1969.

[2] J. Mikusiński, $O$ pochodnych czastkowych i pelnych (On partial and full derivatives, in Polish), Wiadom. Mat. 16 (1973), p. 23-35.

[3] -, On partial derivatives, Bull. Acad. Polon. Sci, Sér. sci. math, astr., phys. 20 (1972), p. 941-944.

[4] -, The Bochner integral, Birkhđuser Verlag Basel und Stuttgart 1978.

Reçu par la Rédaction le 30.08.1980

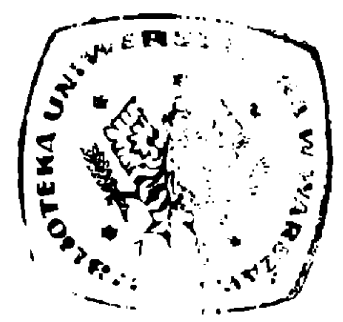

